\title{
Fetal QRS Complex Detection Algorithm for FPGA Implementation
}

\author{
M.I. Ibrahimy ${ }^{1}$, M.B.I. Reaz ${ }^{2}$, F. Mohd-Yasin ${ }^{2}$, T.H. Khoon ${ }^{2}$ and A.F. Ismail ${ }^{1}$ \\ ${ }^{1}$ Kulliyyah of Engineering, International Islamic University, 53100 Kuala Lumpur, Malaysia \\ ${ }^{2}$ Faculty of Engineering, Multimedia University, 63100 Cyberjaya, Malaysia \\ E-mail: ibrahimy@iiu.edu.my
}

\begin{abstract}
An algorithm has been developed for the simultaneous measurement of the fetal and maternal heart rates from the maternal abdominal electrocardiogram during pregnancy and labor for fetal monitoring. The algorithm is based on crosscorrelation, adaptive thresholding and statistical properties in the time domain. Hardware description language - VHDL has been used to implement the algorithm for FPGA implementation. The design is synthesized and fitted into Altera's Stratix EP1S10 using the Quartus II platform. Test case results showed an error percentage of around $\pm 0.3 \%$ and $\pm 0.5 \%$ for the detection of maternal and fetal heart rate respectively.
\end{abstract}

\section{Introduction}

The electrocardiogram (ECG) is the electrical signal produced by the heart and contains the distinctive shape known as the QRS complex. The time between two successive $\mathrm{R}$ peaks of the QRS complex is known as the RR interval and the heart rate (HR) is the reciprocal of the RR interval and expressed in Beat Per Minute (BPM). Electronic fetal heart rate (FHR) monitoring is used to determine if the fetus is free from any complications such as antenatal uteroplacental insufficiency and fetal hypoxia, and to determine the fetal health [1]. Continuous efforts are being made to produce more efficient and accurate methods to extract the fetal RR interval, and gain a better interpretation of the FHR patterns by researchers.

At present, Doppler ultrasound has become a popular technique of monitoring the FHR abdominally but attempts to produce a portable system have not been successful because of its sensitivity to movements [2]. Method utilizing the abdominal electrocardiogram (AECG) has a better prospect for long-term monitoring but requires much signal processing to be done [2][3]. This method is non-invasive and has potential to convey the electro- physiological information, which helps to determine the conditions of the fetus such as stress and acidosis, and uterine activity [3]. A better single-lead method [4] has been adopted and improved to extract the maternal and fetal QRS complexes from the AECG.

The aim of this study is to model a portable system using VHDL - (very high speed hardware description language), which could be used to realize the maternal and fetal QRS complex extraction to measure both the FHR and maternal heart rate (MHR). The VHDL model could be loaded into a Field Programmable Gate array (FPGA) for immediate physical realization. Alternatively, it could be used as the input netlist in a typical design flow, where it would go through synthesis, place-and-route and verification to be developed into a customized device - Application Specific Integrated Circuit (ASIC).

\section{Methods}

\subsection{Maternal QRS complex detection}

The detection of maternal QRS complexes is begun with cross-correlating the signal with an average maternal QRS template. The template is continuously updated with the detection of $\mathrm{R}$ peaks to take into consideration the variation of shape of the maternal QRS complexes in AECG.

The local maxima search routine measures the slope of the cross-correlated output within an R wave search interval. Three sample values and corresponding instants are saved as the local maxima if no maximum is found in the subsequent $20 \mathrm{~ms}$ (assumed to be the minimum fetal QRS duration). This $20 \mathrm{~ms}$ search interval is necessary to avoid taking small spikes on the slopes of the QRS complexes as maxima. One of the maxima is accepted as the $\mathrm{R}$ wave peak by the use of a thresholding technique [5] and comparison to the QRS template. These steps, performed within the validation routine, allow discriminating between the $R$ peak and noise. The length of the search interval is 
initially one second (in fact $1024 \mathrm{~ms}$ for computational simplicity) and it is then continuously updated after the first RR interval measurement. The threshold used in the detection is set initially by assuming a minimum maternal $\mathrm{R}$ peak of $10 \mu \mathrm{V}$ and it is continuously updated based on the levels of both $\mathrm{R}$ peak and noise. Four latest maternal RR intervals are maintained in record for the purpose of checking coincidences of the maternal with the fetal $\mathrm{R}$ waves.

\subsection{Fetal QRS complex detection}

The maternal electrocardiogram (MECG) complex is then subtracted upon detection of a maternal QRS to remove the maternal contribution from the abdominal signal. The MECG template is matched with actual MECG in the abdominal signal by scaling it with the factor obtained from the cross-correlation of abdominal signal with maternal template and autocorrelation of the maternal template. If the crosscorrelation value is greater than the auto-correlation value, then the abdominal signal is multiplied by the factor and MECG template is subtracted, if not, MECG template is multiplied by the factor and subtracted from the abdominal ECG signal.

The detection of the fetal QRS complex is begun with differencing of local maxima and minima [6] on the output of the subtracted signal when the time marker count, which was initiated at the second accepted maternal $\mathrm{R}$ peak, has reached $2048 \mathrm{~ms}$. This duration ensures that the 2 second delayed samples are already within the MECG subtracted region of the signal. A rapid and large deflection between a local maximum and the following local minimum indicates that a fetal beat has occurred. The local maxima search and validation routines are then performed similar to the maternal case. The fetal $\mathrm{R}$ wave search interval is first set at $640 \mathrm{~ms}$ assuming that initially the FHR does not exceed 187 BPM. The validation routine also checks for possible concurrence of the detected maximum with that of the maternal QRS complex.

\subsection{Hardware implementation}

The QRS detection algorithm was initially implemented in Visual $\mathrm{C}++$ because it is simpler and faster to verify the functionality and reliability. Then, the algorithm was implemented in VHDL; where Altera's Quartus II version 4.0 is used as the platform. As a result, for VHDL implementation the algorithm has to be thought of as a structural, behavioral and physical version of the algorithm. The advantage of using Quartus II is that we could synthesize the system into a physically available FPGA, or the built-in simulation device models. The built-in simulation device models emulate the real device with the actual timing and power values. Thus, its performance in terms of timing, speed, power consumption, total logic element counts and functionality could be ascertained. Modifications can be easily performed and its impact to the physical implementation, especially in terms of timing and functionality can be immediately known.

Figure 1 shows a simplified block diagram of the implementation of the system. Basically, the system is categorized into three main blocks, the common, maternal and fetal blocks. The common blocks, shared by both maternal and fetal blocks are the Data Input Block, Memory Register Block, Memory Initialization Block and Main Control Block. Maternal blocks consist of the Maternal Initialization Blocks (XCM1 Block, Inipar1 Block, Maternal Inipar2 Block and IniSub Block), Maternal RR Interval Block, Maternal Validation Block, Maternal Subtraction Block and Maternal Correlation \& Local Maxima Block. Fetal blocks consist of Fetal Initialization Block, Fetal Extraction Block, Fetal Correlation Block, Fetal Local Maxima Search Block, Fetal Template Update Block and Fetal Coincidence Block.

\section{Results and discussion}

\subsection{Simulation results and comparison}

Upon completion of the test case simulation, the system performs a read request to retrieve all the maternal and fetal results. A sample read operation for maternal as well as fetal $\mathrm{R}$ peaks detection is shown in Figure 2 with the initial 4900 sample data. The Quartus simulation result shows that the VHDL models are functioning almost similar to the Visual $\mathrm{C}++$ function. The results for both versions are shown in Table 1 and Table 2. Comparing the maternal and fetal RR interval values (in terms of number of samples between the intervals), the maternal error is consistently less than $0.3 \%$, and the fetal error percentage is within $0.5 \%$.

All the differences are caused by the rounding effect during computation. However, when a fetal peak loss happens, an error rate up to $4 \%$ might be occurred, owing to slightly different search limit implemented in the VHDL. Despite this, the VHDL interpretation of the system displays great similarities to the Visual $\mathrm{C}++$ version. The result in Visual $\mathrm{C}++$ shows encouraging results with the test case, where the fetal $\mathrm{R}$ peak could be detected up to $98 \%$. 


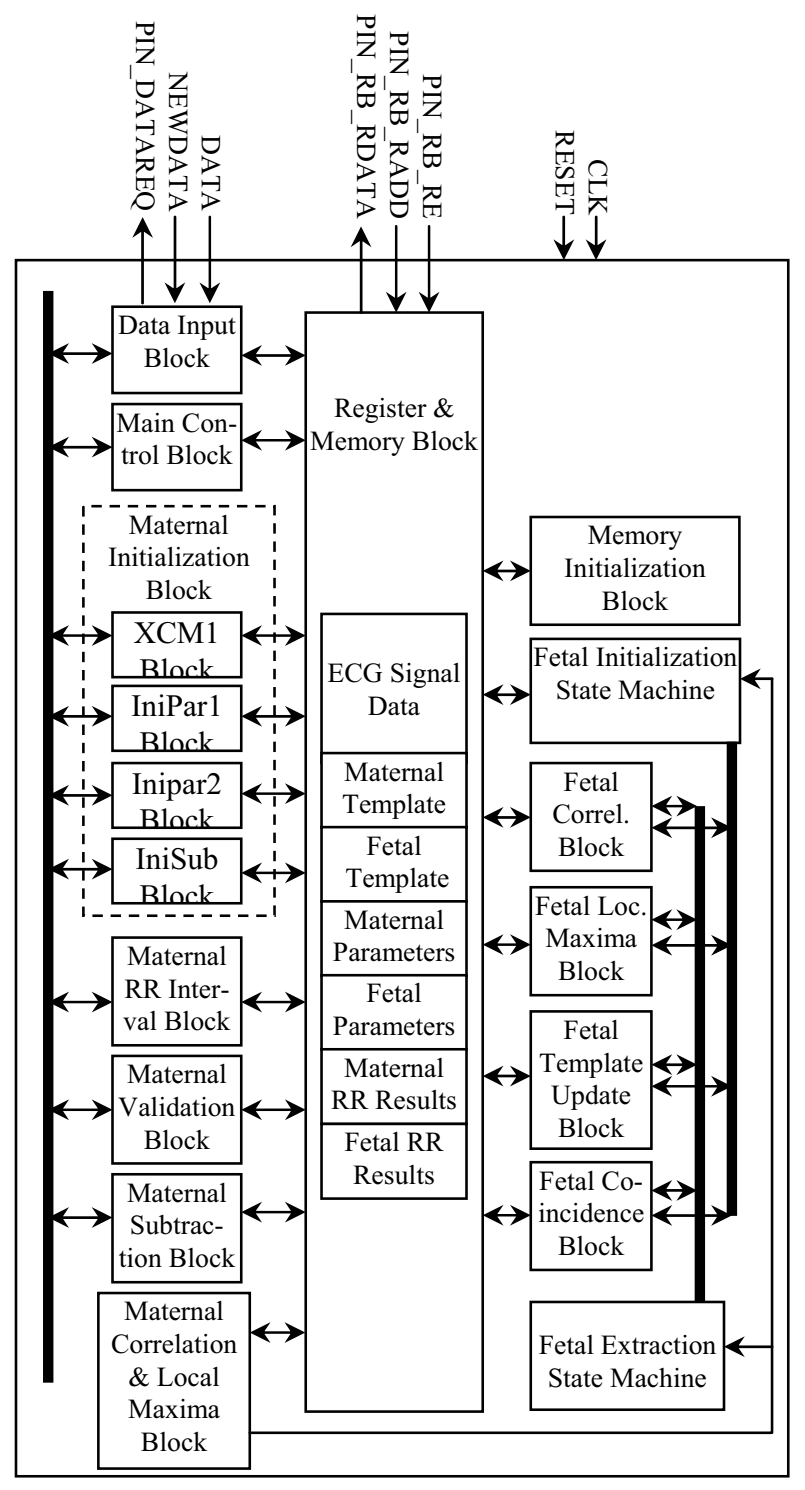

Figure 1. Top level block diagram

\subsection{Resource and device fitting}

Table 3 shows the resources consumed during compilation to fit the system into an Altera's Stratix device. The total resources required are calculated, allocated and then fitted into Altera Stratix EP1S10 device. DSP units are used for the implementation of the accumulator and multiplication between the signal and template points for both the Fetal and Maternal Correlation Blocks.
Table 1. Visual $\mathrm{C}++$ versus VHDL results for maternal $R R$ interval

\begin{tabular}{||c|c|c|c||}
\hline \multirow{2}{*}{ No } & \multicolumn{2}{|c|}{$\begin{array}{c}\text { RR Interval } \\
\text { (Number of Samples) }\end{array}$} & \multirow{2}{*}{$\%$ Diff. } \\
\cline { 2 - 3 } & VC++ & VHDL & \\
\hline 1 & 297 & 297 & $0 \%$ \\
\hline 2 & 299 & 299 & $0 \%$ \\
\hline 3 & 301 & 301 & $0 \%$ \\
\hline 4 & 295 & 295 & $0 \%$ \\
\hline 5 & 296 & 297 & $0.3 \%$ \\
\hline 6 & 294 & 293 & $-0.3 \%$ \\
\hline 7 & 297 & 297 & $0 \%$ \\
\hline 8 & 292 & 292 & $0 \%$ \\
\hline 9 & 299 & 298 & $-0.3 \%$ \\
\hline 10 & 296 & 295 & $-0.3 \%$ \\
\hline 11 & 296 & 295 & $-0.3 \%$ \\
\hline 12 & 290 & 291 & $0.3 \%$ \\
\hline 13 & 289 & 288 & $-0.3 \%$ \\
\hline 14 & 290 & 291 & $0.3 \%$ \\
\hline
\end{tabular}

Table 2. Visual C++ versus VHDL results for fetal RR interval

\begin{tabular}{||c|c|c|c||}
\hline \hline \multirow{2}{*}{ No } & \multicolumn{2}{|c||}{$\begin{array}{c}\text { RR Interval } \\
\text { (Number of Samples) }\end{array}$} & \multirow{2}{*}{$\%$ Diff. } \\
\cline { 2 - 3 } & VC++ & VHDL & \\
\hline 1 & 194 & 194 & $0 \%$ \\
\hline 2 & 197 & 197 & $0 \%$ \\
\hline 3 & 195 & 195 & $0 \%$ \\
\hline 4 & 197 & 198 & $0.5 \%$ \\
\hline 5 & 197 & 196 & $-0.5 \%$ \\
\hline 6 & 198 & 199 & $0.5 \%$ \\
\hline 7 & 196 & 195 & $-0.5 \%$ \\
\hline 8 & 201 & 201 & $0 \%$ \\
\hline 9 & 197 & 197 & $0 \%$ \\
\hline 10 & 312 & 323 & $3.5 \%$ \\
\hline 11 & 290 & 279 & $-3.8 \%$ \\
\hline 12 & 212 & 213 & $0.5 \%$ \\
\hline 13 & 190 & 189 & $-0.5 \%$ \\
\hline 14 & 201 & 201 & $0 \%$ \\
\hline 15 & 188 & 189 & $0.5 \%$ \\
\hline
\end{tabular}

Table 3. Summary of resource fitting

\begin{tabular}{||l|l||}
\hline \hline \multicolumn{1}{|c|}{ Description } & \multicolumn{2}{|c||}{ Total } \\
\hline \hline Device & \multicolumn{2}{|l||}{ EP1S10F780C5 } \\
\hline Netlist Size & \multicolumn{1}{|c||}{10376 Nodes } \\
\hline Usage & $9,633 / 10,570 \quad(91 \%)$ \\
\hline Total Memory & $101,616 / 920,448(11 \%)$ \\
\hline DSP Blocks & $4 / 48$ \\
\hline Total Pins & $346 / 427 \quad(8 \%)$ \\
\hline
\end{tabular}




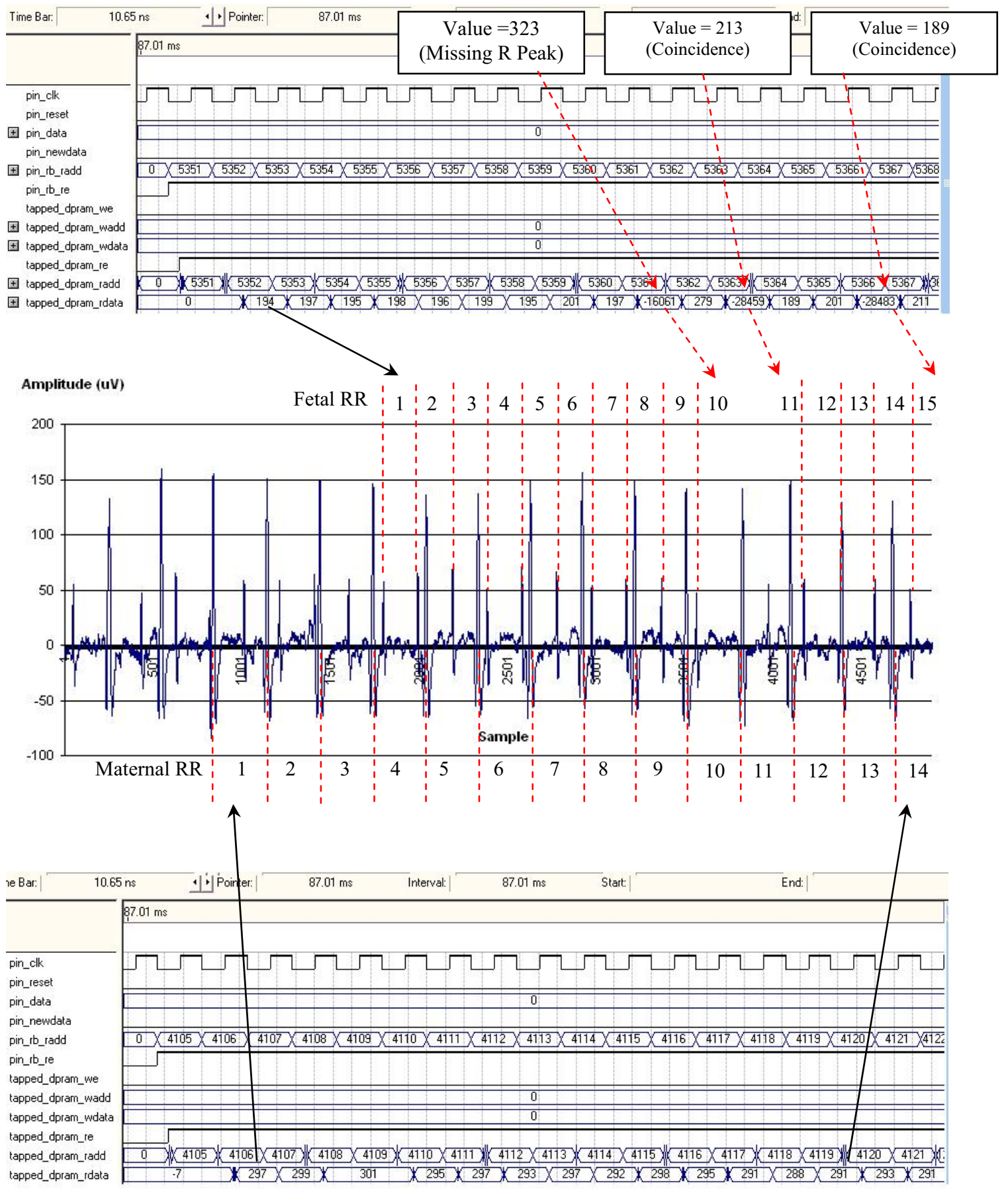

Figure 2: A sample result of maternal and fetal $R$ peaks detection (data: 1-4900)

\section{Conclusion}

The performance achieved for the heart rate measurements from the AECG show that the model can extract both maternal and fetal heart rates utilizing a single-lead configuration. The single-lead feature is desirable from the comfort point of view of the patient especially when the duration of monitoring is long.

Some improvements to the $\mathrm{R}$ peak detection capability of the algorithm would be expected with enhanced procedures such as the normalization of the cross-correlation outputs. The sensitivity of the present 
algorithm to motion artifacts and muscle noise may also be reduced with the incorporation of more rules in its RR interval validation schemes. As expected, a favorable FECG's signal-to-noise ratio is a definitive enhancement in future research. When fully developed, such a system will be a useful tool in the assessment of the fetal condition and its relationship to that of the mother's.

\section{References}

[1] R. K. Freeman, T. J. Garite and M. P. Nageotte, "Fetal Heart Rate Monitoring," Lippincott Williams \& Wilkins: Philadelphia, USA, 2003.

[2] J. A. Crowe, B. R. Hayes-Gill and D. K. James, "Towards Fetal Heart Monitoring at Home Using the Abdominal Fetal Electrocardiogram," in Proc. IEEE Eng. Med. Biol. Soc. $18^{\text {th }}$ Int. Conf., vol. 1, pp. 47-48, 1997.
[3] A. Khamene and S. Negahdaripour, "A New Method for the Extraction of Fetal ECG from the Composite Abdominal Signal," IEEE Trans. Biomed. Eng., vol. 47, pp. 507-516, 2000.

[4] M. I. Ibrahimy, F. Ahmed, M. A. Mohd Ali and E. Zahedi, "Real-Time Signal Processing for Fetal Heart Rate Monitoring", IEEE Trans. Biomed. Eng., vol. 50(2), pp. 258-262, 2003.

[5] J. Pan and W. J. Tompkins, "A Real-Time QRS Detection Algorithm," IEEE Trans. Biomed. Eng., vol. 32, pp. 230-235, 1985.

[6] S. Azevedo and R. L. Longini, "Abdominal-Lead Fetal Electrocardiographic R-Wave Enhancement for Heart Rate Determination," IEEE Trans. Biomed. Eng., vol. 27, pp. 255-260, 1980. 